Bull. Austral. Math. Soc.

Vol. 53 (1996) [469-478]

\title{
WIDTH-DIAMETER RELATIONS FOR PLANAR CONVEX SETS WITH LATTICE POINT CONSTRAINTS
}

\author{
Poh W. Awyong and Paul R. Scott
}

We obtain an inequality concerning the width and diameter of a planar convex set with interior containing no point of the rectangular lattice. We then use the result to obtain a corresponding inequality for a planar convex set with interior containing exactly two points of the integral lattice.

\section{INTRODUCTION}

Let $K$ be a compact, non-empty convex set in $E^{2}$ with minimal width $w(K)=w$ and diameter $d(K)=\delta$. Let $K^{\circ}$ denote the interior of $K$ and let $\Gamma$ denote the integral lattice. A number of results are known concerning the relationship between the width and the diameter of a convex set. The following elegant result was obtained by Scott [3].

THEOREM 1. If $K^{\circ}$ contains no point of $\Gamma$, then $(w-1)(\delta-1) \leqslant 1$ with equality when and only when $K$ is a triangle of diameter $\delta$ and width $w=\delta /(\delta-1)$ (Figure 1).

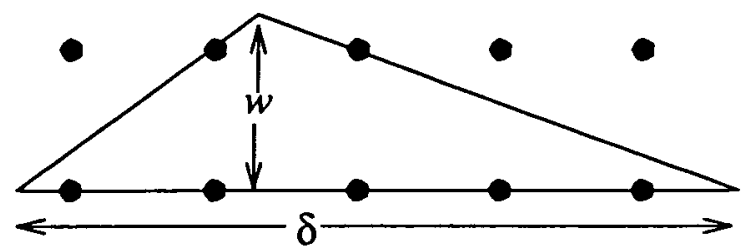

Figure 1.

Theorem 1 has been extended to sets containing exactly one point of $\Gamma$ in the interior [4]. The analogous result is:

Theorem 2. If $K^{\circ}$ contains one point of $\Gamma$, then $(w-\sqrt{2})(\delta-\sqrt{2}) \leqslant 2$; the inequality is best possible.

The purpose of this paper is to generalise Theorem 1 to rectangular lattices and to use the result to obtain analogous inequalities for convex sets containing exactly two points of $\Gamma$ in the interior. Let $\Lambda_{R}(u, v)$ be a rectangular lattice generated by the vectors $(u, 0)$ and $(0, v)$. We prove the following two pretty results:

Received 2nd August, 1995

Copyright Clearance Centre, Inc. Serial-fee code: 0004-9729/96 \$A2.00+0.00. 
Theorem 3. Suppose that $u \leqslant v$ and that $K^{\circ}$ contains no point of $\Lambda_{R}(u, v)$. Then $(w-v)(\delta-u) \leqslant u v$; equality is attained when and only when $K$ is a triangle with diameter $\delta$ and width $w=\delta v /(\delta-u$ ) (Figure 2).

TheOREM 4. If $K^{\circ}$ contains exactly two points of $\Gamma$ then $(w-2)(\delta-1) \leqslant 2$; equality is attained when and only when $K$ is a triangle with diameter $\delta$ and width $w=2 \delta /(\delta-1)$ (Figure 3).

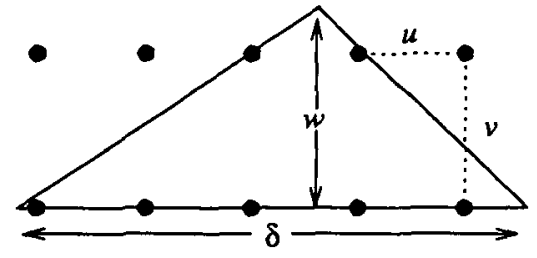

Figure 2.

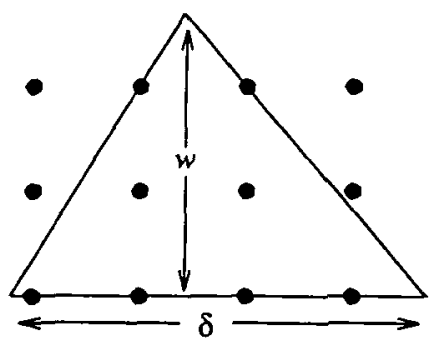

Figure 3.

\section{Three useful lemmas}

We shall denote lines by lower case letters: thus $x$ is a line containing the point $X$ of $\Lambda_{R}(u, v)$. Let the slope of $x$ be $m_{x}$ and let $d(Y, x)$ denote the perpendicular distance from the point $Y$ to the line $x$.

Let $K$ be a set containing no point of $\Lambda_{R}(u, v)$ in its interior. A set for which $(w-v)(\delta-u)$ is as large as possible is called a maximal set. Clearly we may assume that $\delta \geqslant w>v \geqslant u$. We first establish three lemmas which will help us narrow down the possibilities for a maximal set.

We say that a triangle circumscribes a rectangle (or equivalently, a rectangle is inscribed in a triangle) if all vertices of the rectangle lie on the sides of the triangle. Lemma 1 establishes the maximal value of $(w-v)(d-u)$ where $K$ is a triangle circumscribing a fundamental rectangular cell of $\Lambda_{R}(u, v)$. Lemmas 2 and 3 will help us eliminate those cases for which $K$ is not maximal.

LEMMA 1. Let $K$ be a triangle circumscribing a fundamental rectangular cell of $\Lambda_{R}(u, v)$. Then $(w-v)(\delta-u) \leqslant u v$ with equality when and only when the side of the rectangular cell having length $u$ lies on the edge of $K$ with length $\delta$ (Figure 4).

Proof: Let the vertices of $K$ be $X, Y$ and $Z$ and let $\mathcal{C}$ denote the fundamental rectangular cell inscribed in $K$. Without loss of generality, let $X Y$ be the side of $K$ containing two vertices of $\mathcal{C}$. Let $X Y$ have length $b$ and let the altitude from $Z$ to $X Y$ be $h$.

We first let the side of $\mathcal{C}$ with length $u$ lie on the edge $X Y$. Then the area of $K$ is $(1 / 2) b h(=(1 / 2) w \delta)$. The edges of $\mathcal{C}$ partition $K$ into four regions. The area of $K$ 
may therefore be calculated as the sum of the areas of the four component parts (Figure 4).

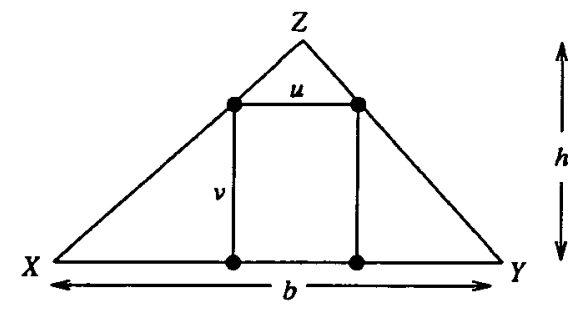

Figure 4.

Hence

$$
\begin{aligned}
\frac{1}{2} w \delta=\frac{1}{2} b h & =\frac{1}{2}(b-u) v+\frac{1}{2}(h-v) u+u v \\
& =\frac{1}{2}(b v+h u),
\end{aligned}
$$

that is,

$$
w \delta=b h=b v+h u .
$$

From the identity $(\alpha+\beta)^{2}=(\alpha-\beta)^{2}+4 \alpha \beta$, we note that the sum of two numbers with a given product is smallest when the difference between them is least. Applying this first to the pair $(b v, h u)$ and then to the pair $(\delta v, w u)$, and noting that $b v-h u \leqslant$ $\delta v-w u$, we have

$$
b v+h u \leqslant \delta v+w u
$$

We thus have

$$
w \delta \leqslant \delta v+w u .
$$

Adding $u v$ to both sides of the inequality gives

$$
(w-v)(\delta-u) \leqslant u v .
$$

Equality is attained here when $X Y=b=\delta$ and $h=w$.

If, on the other hand, the side of length $v$ of $\mathcal{C}$ lies on $X Y$, then by the same argument we obtain $(w-u)(\delta-v) \leqslant u v$. In this case we write

$$
(w-v)(\delta-u)=(w-u)(\delta-v)+(w-\delta)(v-u) .
$$

Since $u \leqslant v$ and $w<\delta$ for triangles, we have

$$
(w-v)(\delta-u)<(w-u)(\delta-v) \leqslant u v .
$$

Hence for circumscribed triangles $K,(w-v)(\delta-u) \leqslant u v$ with equality when and only when the side of $\mathcal{C}$ of length $u$ lies on the edge of $K$ with length $\delta$.

From Lemma 1, we deduce that if $K$ is a maximal set, then $(w-v)(\delta-u) \geqslant u v$. 
Lemma 2. Let $A B C D$ be a fundamental rectangular cell of $\Lambda_{R}(u, v)$ labelled in an anticlockwise direction. Let $\triangle$ be a triangle determined by the lines $a, b$ and $c$ with points $A, B$ and $C$ interior to the edges of $\triangle$ and point $D$ exterior to $\triangle$. Further, let line $c$ containing an edge of $\triangle$ intercept the closed line segment $A D$. Then $(w(\Delta)-v)(d(\Delta)-u)<u v$.

Proof: Let $b . c=P, a . c=Q$ and $a . b=R$. By a suitable rotation of the plane together with a reflection of the set $\triangle$ in the mediator of the segment $A B$, if necessary, we may assume that $m_{b}>m_{c} \geqslant 0$ (see Figure 5).

Suppose first that $\angle Q \leqslant \pi / 2$. Let $c$ make an acute angle $\theta(\neq 0)$ with the line $C D$. Let $V$ be a point on $Q R$ with $B V$ parallel to $P Q$. Then $B V<A B$ and $B V$ is distant $B C \cos \theta<B C$ from $P Q$. We rotate $\triangle$ about $B$ until $P Q$ is parallel to $C D$. Let the rotated triangle be $\triangle^{\prime}$. Clearly $\Delta^{\prime}$ contains no lattice point in its interior and $B$ is the only lattice point on the boundary of $\Delta^{\prime}$. Hence $\Delta^{\prime}$ may be enlarged to a triangle $\triangle^{*}$ inscribing the rectangle $A B C D$. Using Lemma 1 ,

$$
(w(\Delta)-v)(d(\Delta)-u)<\left(w\left(\Delta^{*}\right)-v\right)\left(d\left(\triangle^{*}\right)-u\right) \leqslant u v
$$

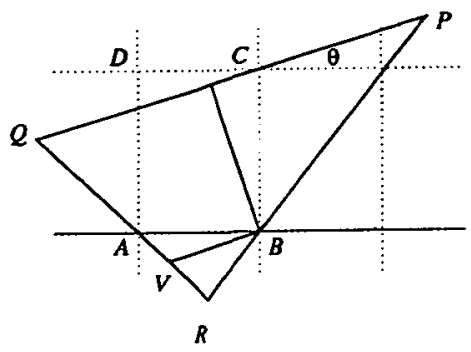

Figure 5.

Now suppose that $\angle Q>\pi / 2$. We consider the following two cases:

CASE (i): $Q$ lies in the closed rectangle $A B C D$. We show that

$$
(w(\Delta)-v)(d(\Delta)-u)<u v .
$$

We first inscribe a rectangle $R_{\Delta}$ in $\triangle$ with side lengths $u^{\prime}<u$ and $v^{\prime}=v$ as follows: Let $b^{\prime}$ be a line parallel to $b$ and distant $v$ from $b$. Since $w>v, b^{\prime}$ intersects $\Delta$ in a line segment $M^{\prime} N^{\prime}$ of length $s>0$ (see Figure 6).

Let $M$ and $N$ be the feet of the perpendiculars from $M^{\prime}$ and $N^{\prime}$ to the line $b$ and let $R_{\Delta}$ be the rectangle with vertices $M, N, N^{\prime}$ and $M^{\prime}$. We shall show that $s<u$. Let $b^{\prime}$ intersect the lines $C D$ and $A D$ in the points $Z$ and $Y$ respectively. Clearly $s<Y Z$. 
We now consider the following two subcases:

(a) If $A B$ has length $u$ and $B C$ has length $v$, we take the coordinates of $B, Z$ and $Y$ to be $(u, 0),(x, v)$ and $(0, y)$ respectively. Hence

$$
\text { Area of } \triangle B Z Y=\frac{1}{2} v \cdot Z Y=\frac{1}{2}\left|\begin{array}{lll}
u & 0 & 1 \\
x & v & 1 \\
0 & y & 1
\end{array}\right|,
$$

that is,

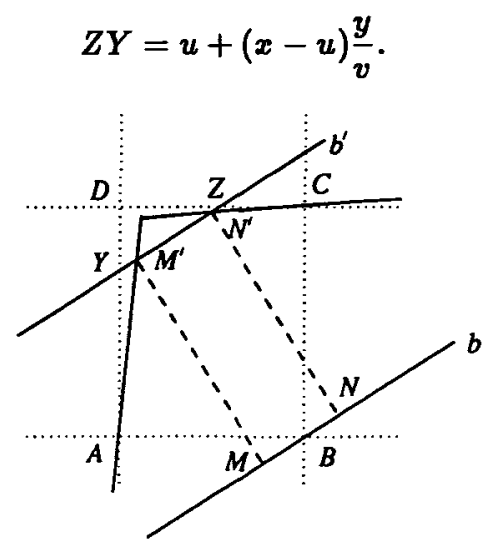

Figure 6.

Now since $x<u$, we have $Z Y<u$. We now rotate $R_{\triangle}$ so that the edge of $R_{\Delta}$ of length $s$ lies on the edge of $A B C D$ of length $u$ and $R_{\triangle}$ is contained in the closed rectangle $A B C D$. The same rotation transforms $\triangle$ to $\triangle^{\prime}$ say. Clearly $\triangle^{\prime}$ contains no interior lattice points and since $s<u$, at least one of $C$ and $D$ lies in the exterior of $\triangle^{\prime}$. Hence $\Delta^{\prime}$ may be enlarged to a triangle $\triangle^{*}$ inscribing the rectangle $A B C D$, and (2) applies immediately.

(b) If now $A B$ has length $v$ and $B C$ has length $u$, we inscribe a rectangle in $\triangle$ with side lengths $\boldsymbol{u}^{\prime}=s$ and $v^{\prime}=v$ as described above. We now let the coordinates of $B, Z$ and $Y$ be $(v, 0),(x, u)$ and $(0, y)$ respectively. Noting that $x<v$, we obtain

$$
Z Y=u+(x-v) \frac{y}{v}<u
$$

By the rotation argument above, we again obtain (2).

CASE (ii): $Q$ lies exterior to the closed rectangle $A B C D$. Let $a$ make an acute angle $\varphi(\neq 0)$ with the line $A D$. Let $T$ be the point on $P Q$ with $B T$ parallel to $Q R$. Now $B T<B C$ and $B T$ is distant $A B \cos \varphi<A B$ from $Q R$. We rotate $\triangle$ clockwise about $B$ until $B T$ lies on the edge $B C$. Let the transformed triangle $\triangle^{\prime}$ have vertices 
$P^{\prime}, Q^{\prime}$ and $R^{\prime}$ corresponding to points $P, Q$ and $R$ respectively. Then clearly $Q^{\prime} R^{\prime}$ is parallel to $A D$. We note also that points $A$ and $C$ are exterior to $\triangle P^{\prime} Q^{\prime} R^{\prime}$. We can now construct a triangle $\triangle^{\prime \prime}$ with vertices $P^{\prime \prime}, Q^{\prime \prime}, R^{\prime \prime}$ such that line $P^{\prime \prime} Q^{\prime \prime}$ is parallel to $P^{\prime} Q^{\prime}$ and contains the point $C$, line $Q^{\prime \prime} R^{\prime \prime}$ is coincident with line $A D$ and line $R^{\prime \prime} P^{\prime \prime}$ is coincident with $R^{\prime} P^{\prime}$. Clearly $\triangle P^{\prime \prime} Q^{\prime \prime} R^{\prime \prime}$ is a triangle of the type described in Case (i). Hence

$$
\begin{aligned}
(w(\Delta)-v)(d(\Delta)-u) & =\left(w\left(\Delta^{\prime}\right)-v\right)\left(d\left(\Delta^{\prime}\right)-u\right) \\
& <\left(w\left(\Delta^{\prime \prime}\right)-v\right)\left(d\left(\Delta^{\prime \prime}\right)-u\right) \\
& <u v
\end{aligned}
$$

This completes the proof of Lemma 2.

Suppose now that $K$ is contained in a triangle satisfying the conditions of Lemma 2. Since $K \subseteq \triangle, w(K) \leqslant w(\Delta)$ and $d(K) \leqslant d(\triangle)$. From Lemma 2, it follows that $K$ is not maximal.

Henceforth we shall use the shorthand notation $L 2(a, b, c)$ to mean:

$K$ is contained in a triangle determined by the lines $a, b, c$ satisfying the conditions of Lemma 2. Hence $K$ is not maximal.

LEMMA 3. Let $A B C D$ be a rectangular cell of $\Lambda_{R}(u, v)$ labelled anticlockwise and let $Q$ be a proper convex quadrilateral determined by lines $a, b, c, d$, with $A$, $B, C$ and $D$ interior to the edges of $Q$ on $a, b, c$ and $d$ respectively. Then amongst all convex sets containing no interior lattice points, a set $K$ contained in $Q$ can not be maximal.

Proof: Since $K \subseteq Q$, it suffices to show that $Q$ is not maximal. Let $a \cdot b=X$, $b . c=Y, c . d=Z$ and $d . a=W$ (Figure 7 ).

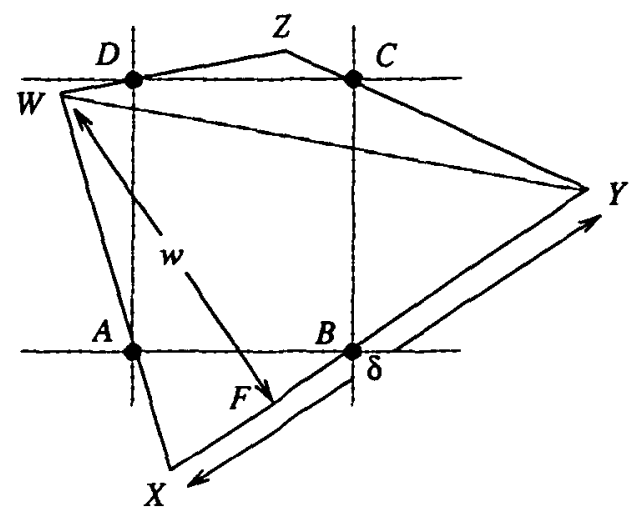

Figure 7. 
We now recall that the diameter of a polygonal set is the maximum distance between a pair of vertices of the polygon. Suppose first that $\delta$ is the length of an edge, $X Y$ say, of $Q$. Without loss of generality, suppose that $W$ is the vertex of $Q$ furthest from $b$. Then $w \leqslant d(W, b)$. Let $\triangle$ be the triangle $X Y W$. Clearly $d(\Delta)=X Y$ and so $w(\Delta)=d(W, b)$ and $w \leqslant w(\Delta)$. But since $\Delta \subset Q, w(\Delta) \leqslant w$. Hence $w=w(\triangle)=d(W, b)$. Since $\triangle$ and $Q$ have the same width and diameter, it suffices to show that $\triangle$ is not maximal. Noting that the edge $W Y$ contains no lattice points, $\triangle$ may be enlarged about the point $X$ to $\Delta^{\prime}=\Delta W^{\prime} X Y^{\prime}$ where $W^{\prime} Y^{\prime}$ contains the point $D$. By a simple variant of Lemma 2 ,

$$
(w(\triangle)-v)(d(\triangle)-v)<\left(w\left(\triangle^{\prime}\right)-v\right)\left(d\left(\triangle^{\prime}\right)-u\right)<u v .
$$

Hence $\triangle$ (and so $Q$ ) is not maximal.

We now suppose that $\delta$ is the length of a diagonal of $Q, W Y$ say. Let $t$ be the width of $Q$ in a direction perpendicular to $W Y$ (see Figure 8). Since the (minimal) width of $Q$ occurs in a direction perpendicular to an edge of $Q$ (see for example [1]), we have $w<t$. Let $W Y$ make an acute angle $\theta$ with $C D$ and let $X Z$ intersect $W Y$ in the point $O$. Now the area of $Q$ is (1/2)t $\delta$. This area is also obtained by adding the areas of the quadrilaterals $O D W A, O B Y C$ to $O C Z D, O A X B$.

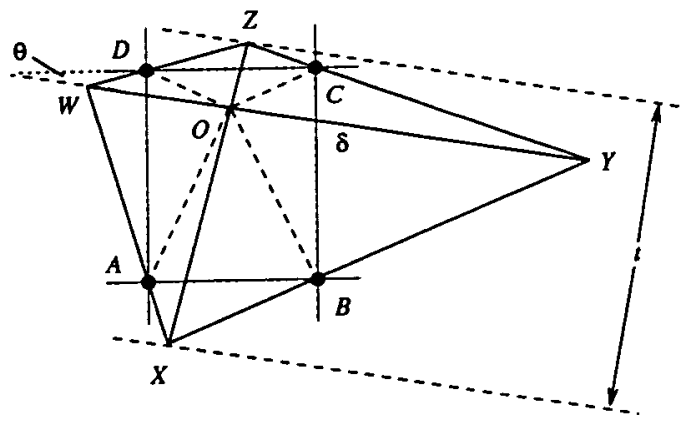

Figure 8.

Suppose first that $A B$ has length $u$ and $B C$ has length $v$. Then we have

$$
\frac{1}{2} t \delta=\frac{1}{2} v \delta \cos \theta+\frac{1}{2} u t \cos \theta .
$$

Hence

$$
t \delta=(t u+\delta v) \cos \theta \leqslant t u+\delta v .
$$

Adding $u v$ to both sides of the inequality and factorising, we have

$$
(t-v)(\delta-u) \leqslant u v .
$$


Since $w<t$, we have

$$
(w-v)(\delta-u)<u v
$$

Hence $Q$ is not maximal.

Now suppose that $A B$ has length $v$ and $B C$ has length $u$. Repeating the above argument, we obtain the corresponding inequality

$$
(w-u)(\delta-v)<u v .
$$

By $(1),(w-v)(\delta-u)<u v$. So again, $Q$ is not maximal.

\section{Proof of Theorem 3}

We now assume that $K$ is a maximal set. We may assume that $\delta \geqslant w>v \geqslant u$. Let the radius of the largest circle inscribed in $K$ be $r$. It is shown in [2] that for any convex set $K$,

$$
(w-2 r) \delta \leqslant 2 \sqrt{3} r^{2}
$$

If $r \leqslant u / 2 \leqslant v / 2$, then

$$
(w-v)(\delta-u)<(w-v) \delta \leqslant(w-2 r) \delta \leqslant 2 \sqrt{3} r^{2} \leqslant 2 \sqrt{3} \cdot \frac{u}{2} \cdot \frac{v}{2}=\frac{\sqrt{3}}{2} u v<u v .
$$

Hence $K$ is not maximal. We may therefore assume that $K$ contains a disk $\mathcal{D}$ of radius $r>u / 2$.

By translating $K$ through a suitable lattice vector, we may bring the centre of $\mathcal{D}$ to lie in $0<y<v$. For easier reference, we list the properties of $\mathcal{D}$ as follows:

D1. $r>u / 2$.

D2. The centre of $\mathcal{D}$ lies in $0<y<v$.

Since $w>v, K^{\circ}$ intercepts one of $y=0$ and $y=v$. Without loss of generality, we may assume that $K^{\circ}$ intercepts $y=0$. Since $K^{\circ}$ contains no point of $\Lambda_{R}(u, v)$, we may assume that $K^{o}$ intercepts $y=0$ between two adjacent lattice points. By translating through a suitable lattice vector we may take these points to be $E(0,0)$ and $F(u, 0)$. Let $G$ and $H$ be the points $(u, v)$ and $(0, v)$ respectively. We shall show that $K$ is a triangle with diameter $\delta$ and width $w=\delta v /(\delta-u)$ (see for example Figure 2).

From D1 and D2, $K^{\circ}$ must intercept one of the edges $E H$ and $F G$. Without losing generality, we may assume that $K^{\circ}$ intercepts $F G$. Hence $K$ lies above a line $f$ with $m_{f}>0$. We now consider the following two cases:

CASE 1: $K$ is bounded by $y=v$. By D1 and D2, lines $e$ and $f$ intersect in the halfplane $y<0$ and $K$ is contained in the triangle $\Delta$ determined by the lines $e, f$ and $y=v$. Since $K^{\circ}$ intercepts $E F, m_{e} \neq 0$. If $m_{e}>0$, then $H$ is exterior to $\triangle$ 
and $L 2(e, f, g)$. We may now assume that $m_{e}<0$ (possibly infinite). In this case, $\Delta$ circumscribes the rectangular cell $E F G H$. By Lemma $1, K$ is maximal when $K$ is the triangle bounded by $y=v$ and the lines $e$ and $f$ with $m_{e}<0$ (possibly infinite) and $m_{f}>0$, and having diameter on the line $y=v$.

CASE 2: $K$ crosses the line $y=v$. We again show that $K$ is not maximal. Suppose that $K$ crosses the line $y=v$ between the adjacent lattice points $X$ and $Y$ on the line $y=v$. Without losing generality, we may assume that $X$ and $Y$ are the points $(k u, v)$ and $((k+1) u, v)$ respectively where $k \geqslant 0$. If $k=0$, then $X=B$ and $Y=G$ and we have $m_{g}<0$ and $m_{h} \neq 0$. If $m_{h}>0$ and $m_{e}<0$, then $K$ is contained in a proper convex quadrilateral $Q$, and by Lemma $3, K$ is not maximal. If $m_{h}<0$ then $L 2(f, g, h)$ or if $m_{e}>0$ then $L 2(f, g, e)$. Finally, if $h$ has infinite slope, $K$ is contained in a triangle circumscribing the rectangle $E F G H$ with the edge $E H$ of length $v$ on $x=0$. By Lemma $1, K$ is not maximal.

We may therefore assume that $X Y \neq G H$. The set $K$ is therefore bounded by lines $x$ and $y$ with $m_{x}>0$. By D1 and D2, $e$ and $f$ intersect in the halfplane $y<0$ and $x$ and $y$ intersect in the halfplane $y>v$. If $m_{f}>m_{x}>0, K$ is contained in a triangle $\Delta$ determined by lines $e, f$ and $x$. Let $g_{f}$ denote the line containing $G$ and parallel to $f$ and let $\pi_{H}$ be the open half plane bounded by $g_{f}$ and containing the point $H$. Since $w(\Delta)>v>d(G, f), e$ and $x$ intersect in a point $Q$ lying in the intersection of the half planes $y \leqslant v$ and $\pi_{H}$. It follows that $K$ is also contained in a triangle $\Delta^{\prime}$ determined by lines $e, f$ and $g_{x}$ where $g_{x}$ is a line containing $G$ and parallel to $x$. Hence $L 2\left(e, f, g_{x}\right)$. If, on the other hand, $m_{x}>m_{f}>0$, then by a similar argument, $K$ is contained in a triangle determined by the lines $x, y$ and $w_{f}$ where $w_{f}$ is the line containing the point $W(k u, 0)$ and parallel to $f$. Hence $L 2\left(y, x, w_{f}\right)$.

This completes the proof of Theorem 3.

\section{Proof of Theorem 4}

Let $K$ now be a set satisfying the conditions of Theorem 4. We may assume that the origin $O$ is one of the lattice points. Let $L\left(z_{1}, z_{2}\right)$ denote the other lattice point contained in $K^{\circ}$. Without loss of generality, we may assume that $z_{1} \geqslant 0$ and $z_{2} \geqslant 0$. By a reflection about the line $y=x$ if necessary, it suffices to consider the cases for which $z_{1} \geqslant z_{2}$. Since $K^{\circ}$ contains no other lattice points, the open line segment $O L$ contains no lattice point. Hence we may assume that $z_{1}$ and $z_{2}$ are relatively prime.

If $z_{1}$ and $z_{2}$ are both odd, we consider the sublattice

$$
\Gamma^{\prime}=\{(x, y): x+y \equiv 1 \quad(\bmod 2)\} \text {. }
$$

Clearly $O \notin \Gamma^{\prime}, L \notin \Gamma^{\prime}$ and $K^{\circ}$ contains no point of $\Gamma^{\prime}$. By Theorem 3, we have

$$
(w-\sqrt{2})(\delta-\sqrt{2}) \leqslant 2 \text {. }
$$


However,

$$
\begin{aligned}
(w-2)(\delta-1)-(w-\sqrt{2})(\delta-\sqrt{2}) & =w(\sqrt{2}-1)+\delta(\sqrt{2}-2) \\
& \leqslant \delta(\sqrt{2}-1)+\delta(\sqrt{2}-2) \\
& =\delta(2 \sqrt{2}-3)<0
\end{aligned}
$$

It follows that $(w-2)(\delta-1)<(w-\sqrt{2})(\delta-\sqrt{2}) \leqslant 2$. Hence $K$ is not maximal.

If say, $z_{1}$ is odd and $z_{2}$ is even, we consider the sublattice

$$
\Gamma^{\prime}=\{(x, y): x=n, y=2 m+1, m, n \in Z\}
$$

Clearly $O \notin \Gamma^{\prime}, L \notin \Gamma^{\prime}$ and $K^{\circ}$ contains no point of $\Gamma^{\prime}$. By Theorem 3, we have

$$
(w-2)(\delta-1) \leqslant 2 .
$$

Equality occurs when and only when $K$ is a triangle with diameter $\delta$ and width $w=$ $2 \delta /(\delta-1)$ as shown in Figure 3.

\section{REFERENCES}

[1] P.R. Scott, 'A lattice problem in the plane', Mathematika 20 (1973), 247-252.

[2] P.R. Scott, 'Two inequalities for convex sets in the plane', Bull. Austral. Math. Soc. 19 (1978), 131-133.

[3] P.R. Scott, 'Two inequalities for convex sets with lattice point constraints in the plane', Bull. London Math. Soc. 11 (1979), 273-278.

[4] P.R. Scott, 'On planar convex sets containing one lattice point', Quart. J. Maths. Oxford Ser. (2) 36 (1985), 105-111.

\footnotetext{
Department of Pure Mathematics

The University of Adelaide

South Australia 5005

Australia

e-mail: pawyong@maths.adelaide.edu.au

pscott@maths.adelaide.edu.au
} 